\title{
SIMPLIFIED PROOFS OF SOME BASIC THEOREMS FOR QUASIREGULAR MAPPINGS
}

\author{
MARTTI I. PESONEN
}

\section{Introduction}

In what follows $f$ will always denote a non-constant $n$-dimensional quasiregular mapping of a domain $G \subset \boldsymbol{R}^{n}$ into $\boldsymbol{R}^{n}$. We recall that the branch set $B_{f}$ is the set of those points in $G$ at which $f$ is not locally homeomorphic, and that $N(y, f, A)$ is the number of all points in the set $f^{-1}(y) \cap A$. Our notation and terminology is adopted from [1].

The purpose of this paper is to present new simplified proofs for the following well-known theorems in the theory of quasiregular mappings.

1.1. Theorem. The condition $(N)$ is satisfied, i.e., if $A \subset G$ and $m(A)=0$, then $m(f A)=0$. Moreover $m\left(f B_{f}\right)=0$.

\subsection{Theorem. The transformation formula}

$$
\int_{\boldsymbol{E}}(h \circ f) J_{f} d m=\int_{\boldsymbol{R}^{n}} h(y) N(y, f, E) d m(y)
$$

holds whenever $h: \boldsymbol{R}^{n} \rightarrow[0, \infty]$ and $E \subset G$ are measurable.

1.3. Theorem. For a.e. $x \in G, J_{f}(x) \neq 0$. Consequently $m\left(B_{f}\right)=0$.

Rešetnjak's original proof for the condition $(N)$ does not make use of the fact that $f$ is discrete and open. In the present proof these properties of $f$ play an essential role. It should be noted that 1.1 is not needed in proving the discreteness and openness of $f$ (see [4]).

Theorem 1.2 is a direct consequence of the proof of Theorem 1.1. Earlier the transformation formula was obtained by the use of a general theorem [3, p. 364] the proof of which requires a heavy machinery of algebraic topology.

The original proof $[1,8.2]$ of Theorem 1.3 is based on the $K_{I}$-capacity inequality. Our proof instead is, based on the use of the $K_{0}$-path family inequality and Poleckii's lemma. 


\section{The proofs of Theorems 1.1 and 1.2}

Because $f$ is continuous and a.e. differentiable, it is a basic fact of real analysis that if $f$ is injective, then

$$
m(f E) \geqq \int_{E} J_{f} d m
$$

for every Borel set $E$ in $G$. In fact, the equality holds in (2.1). This is a consequence of the following result, which is obtained by a $C^{1}$-approximation.

2.2. Proposition. For every Borel set $E$ in $G$

$$
m(f E) \leqq \int_{E} J_{f} d m
$$

Proof. We first show that $n$-intervals in $G$ can be approximated by $n$-intervals whose boundaries $f$ maps into null-sets. To do this, fix a closed $n$-interval $Q$ in $G$ and let $\varepsilon$ be positive. Let $Q^{\prime}$ be a closed $n$-interval in $G$ so that $Q \subset \operatorname{int} Q^{\prime}$ and $m\left(Q^{\prime} \backslash Q\right)<\varepsilon$. If $m\left(f \partial Q_{0}\right)>0$ for every $n$-interval $Q_{0}, Q \subset Q_{0} \subset Q^{\prime}$, then there is a positive number $p$ and a sequence of $n$-intervals $Q_{i}, Q \subset Q_{i} \subset Q^{\prime}$, with disjoint boundaries such that $m\left(f \partial Q_{i}\right) \geqq p$ for every $i$. But this is impossible, since

where

$$
\sum_{i} m\left(f \partial Q_{i}\right)=\int_{R^{n}} \sum_{i} \chi_{f \partial Q_{i}} d m \leqq N\left(f, Q^{\prime}\right) m\left(f Q^{\prime}\right)<\infty,
$$

$$
N\left(f, Q^{\prime}\right)=\sup \left\{N\left(y, f, Q^{\prime}\right) \mid y \in \boldsymbol{R}^{n}\right\} .
$$

Hence, $m\left(f \partial Q_{0}\right)=0$ for some $n$-interval $Q_{0} \supset Q$ with $m\left(Q_{0} \backslash Q\right)<\varepsilon$.

Let $\varepsilon_{1}$ be positive. It follows from the definition of the Lebesgue measure and from the approximation result mentioned above, that since $J_{f}$ is locally integrable ( $f$ is $A C L^{n}$ ), there exists a sequence of closed $n$-intervals $Q_{i} \subset G$ with $m\left(f \partial Q_{i}\right)=0$, such that $E \subset \cup_{i} Q_{i}$ and

$$
\sum_{i} \int_{Q_{i}} J_{f} d m \leqq \int_{E} J_{f} d m+\varepsilon_{1}
$$

On the other hand, $m(f E) \leqq \sum_{i} m\left(f Q_{i}\right)$, so that it remains to show that the proposition holds for any closed $n$-interval $Q$ in $G$ satisfying $m(f \partial Q)=0$. By [5; 27.7] there are $C^{1}$-mappings $f_{1}, f_{2}, \ldots$, which converge $c$-uniformly to $f$ and whose Jacobians $J_{f_{i}}$ converge to $J_{f}$ in $L_{\mathrm{loc}}^{1}$. Set $\chi=\chi_{f Q}$ and $\chi_{j}=\chi_{f_{j} Q}$. In order to show that $\chi_{j} \rightarrow \chi$ a.e., we first pick a point $y$ in $f Q \backslash f \partial Q$ and note that the local topological degree $\mu$ satisfies $\mu\left(y, f_{j}\right.$, int $\left.Q\right)=\mu(y, f$, int $Q)>0$ for $j \geqq j_{0}$, since the convergence is $c$-uniform and $f$ is sense-preserving. Hence $y \in f_{j} Q$ if $j \geqq j_{0}$, and $\chi_{j}(y) \rightarrow \chi(y)$. Outside $f Q$ the convergence $\chi_{j} \rightarrow \chi$ is obvious, so that $\chi_{j} \rightarrow \chi$ 
a.e. in $\boldsymbol{R}^{n}$. To complete the proof we apply Fatou's lemma, and get

$$
m(f Q)=\int_{R^{n}} \chi d m \leqq \varliminf_{j \rightarrow \infty} \int_{R^{n}} \chi_{j} d m=\varliminf_{j \rightarrow \infty} m\left(f_{j} Q\right) \leqq \varliminf_{j \rightarrow \infty} \int_{Q}\left|J_{f_{j}}\right| d m=\int_{Q} J_{f} d m,
$$

where the latter inequality comes from elementary calculus.

Theorem 1.1 is an immediate consequence of 2.2 ; for the last statement, recall that $J_{f}=0$ a.e. in $B_{f}$. Since $f$ satisfies the condition $(N)$, it is obvious that (2.1) and 2.2 hold in fact for any measurable set $E$ in $G$.

To prove the transformation formula, we first consider the case that $h=$ $\sum_{j=1}^{\infty} a_{j} \chi_{B_{j}}(\geqq 0)$ is a simple Borel function. Since $m\left(f B_{f}\right)=0$ and $J_{f}=0$ a.e. in $B_{f}$, we may assume that $E$ does not meet $B_{f}$. Let $E_{1}, E_{2}, \ldots$ be a measurable partition of the set $E$, such that each $E_{k}$ is contained in a domain on which $f$ is injective. Then

$$
\begin{gathered}
\int_{E}(h \circ f) J_{f} d m=\sum_{j, k} a_{j} \int_{E_{k} \cap f^{-1} B_{j}} J_{f} d m=\sum_{j, k} a_{j} m\left(f E_{k} \cap B_{j}\right) \\
=\int_{R^{n}} \sum_{j} a_{j} \chi_{B_{j}} \sum_{k} \chi_{f E_{k}} d m=\int_{R^{n}} h N(\cdot, f, E) d m .
\end{gathered}
$$

Finally, if $h \geqq 0$ is measurable, then there is an increasing sequence $\left(h_{i}\right)$ of simple Borel functions, which converge to $h$ a.e.. It follows from (2.1) that also $h_{i} \circ f \rightarrow h \circ f$ a.e. outside the sct $\left\{x: J_{f}(x)=0\right\}$, and hence Theorem 1.2 follows by the monotonic convergence theorem.

\section{Proof of Theorem 1.3}

From 1.2 it follows easily (see $[1 ; 3.2])$ that

$$
M(\Gamma) \leqq K_{0}(f) N(f, A) M(f \Gamma)
$$

if $\Gamma$ is a path family in a Borel set $A \subset G$, and $N(f, A)<\infty$. This path family inequality and Poleckii's lemma 3.2 will be needed in the proof of 1.3 .

3.2. Lemma [2]. If $\Gamma_{0}$ is the family of all closed paths in $G$ on which $f$ is not absolutely precontinuous, then $M\left(f \Gamma_{0}\right)=0$.

Recall that $f$ is called absolutely precontinuous on $\gamma$ if $f \circ \gamma$ is rectifiable and if the reparametrization $\gamma^{*}$ of $\gamma$ with

$$
f \circ \gamma^{*}=(f \circ \gamma)^{0}
$$

is absolutely continuous. Here $\alpha^{0}$ denotes the parametrization of $\alpha$ by means of path length.

Proof of 1.3. Suppose that $J_{f}=0$ in a set of positive measure. This set then contains a Borel set $B$ of positive measure such that $B \subset Q$, where $Q$ is a closed $n$-interval in $G$, and that $f$ is differentiable and $f^{\prime}(x)=0$ for every $x \in B$. Let 
$\Gamma_{B}$ be the family of all closed intervals $\gamma$ in $Q$ parallel to $e_{1}=(1,0, \ldots, 0)$ with $\int_{\gamma} \chi_{B} d s>0$. Fubini's theorem implies that $M\left(\Gamma_{B}\right)>0$. By 31

$$
0<M\left(\Gamma_{B}\right) / K_{0}(f) N(f, Q) \leqq M\left(f \Gamma_{B}\right),
$$

so that according to Poleckii's lemma there is a path $\gamma \in \Gamma_{B}$ such that $\gamma^{*}$ is absolutely continuous. Thus

$$
0<\int_{\gamma} \chi_{B} d s=\int_{0}^{l(f \circ \gamma)}\left(\chi_{B} \circ \gamma^{*}\right)\left|\gamma^{* \prime}\right| d m_{1}=\int_{\gamma^{*} 1_{B}}\left|\gamma^{* \prime}\right| d m_{1},
$$

and consequently $m_{1}\left(\gamma^{*-1} B\right)>0$. On the other hand, for $m_{1}$-a.e. $t \in \gamma^{*-1} B$,

$$
1=\left|(f \circ \gamma)^{0^{\prime}}(t)\right|=\left|\left(f \circ \gamma^{*}\right)^{\prime}(t)\right|=\left|f^{\prime}\left(\gamma^{*}(t)\right) \gamma^{* \prime}(t)\right|=0,
$$

which is clearly absurd. Therefore $J_{f} \neq 0$ a.e. Since $J_{f}=0$ a.e. in $B_{f}$, it follows that $m\left(B_{f}\right)=0$.

3.3. Remark. In [2] Poleckii uses 3.2 to prove his celebrated $K_{I}$-path family inequality. In his proof he needs the result 1.3, whose original proof requires the use of the $K_{I}$-capacity inequality. This latter inequality is quite hard to prove, and, on the other hand, is a special case of the $K_{I}$-path family inequality. It is therefore important to have a proof for 1.3 which does not make use of the $K_{I}$-capacity inequality.

S. Rickman has pointed out that it would also be possible to modify the proof of the $K_{I}$-path family inequality in such way that 1.3 is not needed in the proof.

\section{References}

[1] Martio, O., S. RickMan, and J. VÄISÄLÄ: Definitions for quasiregular mappings. - Ann. Acad. Sci. Fenn. Ser. A I Math. 448, 1969, 1-40.

[2] Poleckil̆, E. A.: The method of modul for nonhomeomorphic quasiconformal mappings. Mat. Sb. (N.S.) 83 (125), 1970, 261-272 (Russian).

[3] Rado, T., and P. V. Reichelderfer: Continuous transformations in analysis. - Die Grundlehren der mathematischen Wissenschaften 75, Springer-Verlag, Berlin-GöttingenHeidelberg, 1955.

[4] RICKMAN, S.: Lectures on quasiregular mappings. - To appear.

[5] VÄISÄL ̈̈, J.: Lectures on $n$-dimensional quasiconformal mappings. - Lecture Notes in Mathematics 229, Springer-Verlag, Berlin-Heidelberg-New York, 1971.

University of Helsinki

Department of Mathematics

SF-00100 Helsinki 10

Finland

Received 4 March 1983 